\title{
Le rôle de la Société du Canal de Provence dans la gestion des ressources en eau de la région provençale
}

\author{
SCP role in management of water resources in the provençal region
}

\author{
par J. Plantey \\ Directeur des Services Techniques, SCP \\ L. Reig \\ Directeur adjoint, chargé de l'aménagement, SCP
}

Today, the Provençal Region has largely overcome the risk of water shortages. Indeed, 40 years ago all the parties affected by a policy of good management of the region water resources as part of the general interest decided to work together in order to institute original solutions. These have proved pertinent and effective.

The creation of the reserves needed to guarantee the satisfaction of water requirements associated with the development of all sectors of activity (urban, industrial and agricultural) was allied to the construction of major hydroelectric production facilities. The regional and local authorities involved together decided to execute and manage the facilities for conveying these resources to the points of consumption. To satisfy this common aim, they created the "Société du Canal de Provence et d'Aménagement de la Région Provençale (SCP) ", a regional development company within the meaning of French rural legislation, an original structure in many respects, particularly in terms of its status and its mission.

All the actions undertaken by SCP in the framework of its public service mission result from a coherent and concerted policy founded on the common interest, fundamental principle of a transparent and quality public service run to satisfy economic performance and efficiency criteria. The economic management of the water resource applied on the scale of the schemes designed, constructed and operated by SCP is the combination and the result of a series of technical, financial, economic and management arrangements. Amid all the interrogations, reflections and recommendations encountered on the lasting responsibility of users, deprivation of public services, etc. which are at the heart of current debates, SCP, after 40 years of managing a water resource to the satisfaction of multiple users, stands out as an original solution which contributes to the on-going process of reflection.

\section{LA PROVENCE : TERRE DE CONTRASTE}

Bien que bénéficiaire d'une pluviométrie globalement convenable, la Provence est pénalisée par son climat qui se caractérise par l'alternance de très longues périodes de sécheresse absolue et de précipitations brutales qui s'écoulent à torrents ou s'infiltrent dans les massifs calcaires sans pour autant créer des sources pérennes fiables.

De plus, la morphologie et la géologie de la Provence, et plus particulièrement celles des Bouches-du-Rhône et du Var, ont toujours posé aux collectivités des problèmes d'approvisionnement en eau. Cette région est en effet formée d'une série de chaines calcaires, de direction générale EstOuest, parallèles à la côte méditerranéenne, entre lesquelles sont insérées de petites plaines alluviales.

Paradoxalement, alors que plus au Nord le massif alpin est riche en eau, ces chaînes montagneuses constituent une barrière qui repousse vers l'Ouest les eaux de la Durance et du Verdon et éloigne ainsi les ressources des zones côtières où s'expriment les besoins les plus forts.

De ce fait, depuis l'Antiquité, la gestion de l'eau a été un facteur clé de la civilisation provençale et de son développement.

Confrontée à cette problématique, dont l'ampleur ne s'accommodait guère de solutions à échelle individuelle et concernait l'ensemble de la collectivité, la Provence a fait œuvre, très tôt, d'aménagement du territoire, dans sa volonté de s'affranchir durablement des risques de pénurie et valoriser pleinement les ressources mobilisées.

Ainsi, la Provence présente aujourd'hui toute une gamme de réalisations résultant d'une accumulation séculaire de travaux progressivement réalisés, remodelés, transformés, déve- 
loppés avec toutes les phases que cela implique de dispositions novatrices, mais aussi d'échecs surmontés et de réajustements permanents aux nécessités de l'époque.

\section{II $\square$ MOBILISER LA RESSOURCE EN EAU : MILLE ANS DE PROJETS COLLECTIFS}

Dès l'occupation romaine, de nombreux ouvrages hydrauliques ont été réalisés, mais la fonction essentielle de ces aqueducs était d'apporter l'eau aux villes pour les besoins des populations.

Les invasions qui ont suivi la chute de l'Empire Romain ont souvent conduit à leur dégradation mais, dès le début de l'actuel millénaire, les problèmes de maîtrise des eaux ont préoccupé de nouveau les populations provençales afin de contribuer à la protection des terres contre les inondations, la desserte en eau des populations et la satisfaction des besoins des activités agricoles et économiques.

La croissance considérable de la population aux XIle et XIIIe siècles a ainsi contraint les territoires de Provence à rechercher des ressources nouvelles en eau, en énergie et en produits alimentaires.

Pendant des siècles, les besoins des populations furent en effet satisfaits par les modestes ressources locales, mais l'idée d'utiliser les eaux du bassin de la Durance pour desservir convenablement la région a germé très tôt dans les esprits.

Ainsi, les communautés situées à proximité de la Durance se préoccupèrent d'utiliser au mieux cette ressource. Compte tenu de la pente élevée de la rivière, la première utilisation envisagée fut destinée à la production d'énergie. La dérivation se faisait alors avec un canal à pente très faible et très rapidement on a ainsi disposé d'une chute suffisante pour faire tourner les moulins à moudre ou à fouler. Les agriculteurs constatèrent très vite tout le bénéfice qu'ils pouvaient tirer de l'irrigation et les usiniers leur permirent d'utiliser les eaux pour l'arrosage " du samedi soir, soleil couché, au lundi matin, soleil levé ". Le jour du Seigneur étant aussi celui de l'irrigant, c'est ainsi que furent créés le Canal de l'Hôpital dans le Vaucluse au XIle siècle, le Béal du Moulin de Sénas au XIIle siècle.

Dès 1507, François 1er accordait alors à la Maison d'Oppède une concession pour la création d'un " Canal de Provence".

Quelques dérivations se poursuivirent ainsi au cours des ans, mais il fallut attendre le milieu du XVIe siècle pour qu'Adam de Craponne se lance dans une œuvre beaucoup plus importante et audacieuse en dérivant les eaux de la Durance en dehors de son bassin versant direct pour les transférer en Crau, vers Salon de Provence, avec pour principaux objectifs : produire de l'énergie et développer les irrigations.

Mais le promoteur avait surestimé ses capacités financières et sous-estimé la faculté de ses concitoyens à contribuer financièrement à un investissement qui, par ailleurs, était pour eux tout à fait rentable. Il mourut ruiné, comme ce fut d'ailleurs le cas pour bon nombre de promoteurs d'ouvrages hydrauliques.

A la fin du XVIIIe siècle, et surtout dans la deuxième moitié du XIXe siècle, les canaux se sont multipliés en conservant l'objectif énergétique, mais avec une destination agricole qui devenait de plus en plus importante.

Ainsi, au fil des siècles, s'est développée une série d'ou- vrages à buts multiples assurant la meilleure valorisation des eaux de la Durance et de ses affluents.

Mais amener vers Aix, Marseille et Toulon les eaux de la Durance et du Verdon, son principal affluent, allait, par la suite, devenir une préoccupation majeure et permanente des collectivités provençales, ainsi d'ailleurs que de toute une série d'ingénieurs promoteurs.

Ainsi, dès 1559, Adam de Craponne proposait à la Ville d'Aix un projet qui ne put être réalisé, faute de moyens financiers. Pendant trois siècles, ce projet fut repris une dizaine de fois, en vue de l'alimentation conjointe d'Aix et de Marseille. et des travaux furent même amorcés, rapidement bloqués par manque de moyens financiers et techniques suffisants, ou d'accords effectifs entre les villes d'Aix et de Marseille.

A la suite de la sécheresse exceptionnelle de 1829 et de la terrible épidémie de choléra en 1834 , la ville de Marseille décidait en 1835 de réaliser le canal de Marseille, laissant le soin à la ville d'Aix de créer un second canal pour s'alimenter.

Pour Aix, l'élaboration et l'approbation de projets d'un canal dérivé du Verdon s'étalent de 1837 à 1863 et sa construction s'achève en 1875 après la défaillance de plusieurs concessionnaires. Reprise par la ville d'Aix, la concession finit par être rachetée en 1927 par le Département des Bouches-du-Rhône, et en définitive transférée, en 1963, à la Société du Canal de Provence et d'Aménagement de la Région Provençale.

\section{III — LA MAÎTRISE DE L'EAU EN PROVENCE : UNE IMPÉRIEUSE NÉCESSITÉ DU XXE S.}

Malgré la mise en place de ces importants aménagements au cours du XIXe siècle et l'adoption des lois de 1907 et 1923 autorisant la dérivation des eaux du bassin de la Durance, des pénuries dramatiques se produisaient à l'étiage et le département du Var et l'Est des Bouches-du-Rhône restaient privés d'accès à ces ressources en eau.

Aussi, alors que le gisement hydraulique du bassin était considérablement sous-exploité, l'ensemble des besoins en eau des populations et des activités agricoles et économiques ne pouvait être satisfait par le fait principal que la régularisation des prélèvements n'était pas assurée.

Les travaux d'aménagement à entreprendre représentaient des enjeux considérables.

L'expérience du passé avait montré que de tels aménagements étaient promis à la ruine si des dispositions financières particulières n'étaient pas prises pour faire face aux charges intercalaires liées à la très longuée durée de retour des investissements ; et si une politique rigoureuse d'entretien et de gestion des ouvrages n'était pas systématiquement appliquée, pour garantir leur pérennité. En tenant compte de ces enseignements, des dispositions particulières ont été prises à la fin des années 50 , visant à mettre en place une gestion globale et cohérente des ressources en eau.

En 1952 fut créé le Service Spécial du Bassin de la Durance à l'initiative du ministère de l'Agriculture, pour assurer les liaisons nécessaires entre ce département ministériel et Electricité de France (EDF) qui s'était vue confiée la mission de valorisation énergétique de la Durance et du Verdon (loi du 5 janvier 1955).

Dans ce dernier cadre EDF réalisa une série de barrages à buts multiples sur la Durance et le Verdon dans lesquels les 
volumes de stockage nécessaires à la satisfaction des besoins en eau d'une grande partie du territoire régional furent réservés, notamment dans les retenues de Serre-Ponçon sur la Durance et Sainte-Croix sur le Verdon.

En parallèle, le SSBD était chargé d'un projet devant répondre aux besoins de trois importantes collectivités locales : les départements des Bouches-du-Rhône, du Var et la ville de Marseille. Il convenait, pour ce faire, de réaliser une infrastructure hydraulique permettant d'assurer le transport des eaux brutes dérivées du Verdon, vers les zones de Toulon, Aix et Marseille, et de mettre en place un ensemble de réseaux de distribution pour les usagers agricoles, urbains et industriels.

Un tel aménagement à buts multiples présentait pour tous les usagers un intérêt incontestable car il permettait d'obtenir leur desserte commune dans des conditions économiques acceptables, alors que la construction d'un ouvrage consacré à la seule desserte de l'un quelconque de ces besoins (agricole, rural, urbain ou industriel) eût été beaucoup trop onéreuse. Ce groupement permettait, en outre, de faire bénéficier les irrigations agricoles de tarifs préférentiels sans, pour autant, léser les autres catégories d'usagers.

Pour assurer l'alimentation en eau de l'agriculture, des villes et des industries des Bouches-du-Rhône et du Var, une structure opérationnelle devait être créée. Après avoir envisagé la création d'un syndicat interdépartemental regroupant les collectivités concernées pour assurer la maîtrise d'ouvrage du projet, cette solution fut abandonnée en raison de la lourdeur administrative à laquelle elle conduisait.

\section{IV — LA SOCIÉTÉ DU CANAL DE PRO- VENCE : L'INSTRUMENT D'UNE POLI- TIQUE RÉGIONALE DE L'EAU}

Dans le cadre d'une politique active d'aménagement du territoire, le ministère de l'Agriculture souhaitait mettre en place un organisme adapté aux besoins régionaux. Il proposa la constitution d'une société anonyme d'économie mixte comprenant à l'origine les départements des Bouches-duRhône, du Var, la ville de Marseille, rejoints par les deux départements alpins, la profession agricole et les banques chargées du développement agricole et de l'équipement du territoire (CNCA et CDC) et ayant le statut de Société d'Aménagement Régional défini par une loi de 1951, reprise en 1992 dans le Code Rural.

Ainsi fut créée, le 11 juillet 1957, la Société du Canal de Provence et d'Aménagement de la Région Provençale (SCP) qui reçut de la part de l'Etat une mission générale d'aménagement et de service public pour gérer les réserves en eau du Verdon et réaliser l'ouvrage Canal de Provence, mais aussi les autres ouvrages hydrauliques qui s'avéreraient nécessaires au développement de la région provençale. Le décret de concession d'Etat du 15 mai 1963 en arrête les dispositions d'ensemble.

L'Etat et les collectivités locales, associés étroitement dans ce dispositif, ont pu progressivement définir les conditions de financement des ouvrages, trouver les moyens pour faire face convenablement aux charges intercalaires, arrêter les dispositions permettant d'assurer une mission de service public avec un maintien en parfait état opérationnel des ouvrages réalisés au fil des ans.

L'œuvre commune ainsi engagée, sous l'impulsion de l'Etat, par les collectivités territoriales et les acteurs locaux était prémonitoire en répondant par avance à des concepts, préoccupations et politiques développés plus de vingt ans plus tard dans plusieurs domaines :

- la responsabilisation de l'ensemble des acteurs, et en particulier des consommateurs, des collectivités locales et de l'organisme gestionnaire,

- l'action conjointe et concertée prônée par les politiques contractuelles de planification et de coopération décentralisée entre l'Etat et les Régions (contrat de plan Etat-Région), - la gestion économe de l'eau, patrimoine commun, dans le cadre de l'intérêt général et dans une perspective à long terme.

C'est à la lumière de cette ambition qu'est analysé ci-après le projet Canal de Provence.

\section{LE CANAL DE PROVENCE : INTÉRÊT GÉNÉRAL ET DÉVELOPPEMENT DURABLE}

L'importance d'une gestion patrimoniale des ressources naturelles, condition nécessaire de développement durable, est aujourd'hui clairement affirmée par l'article 1 de la loi sur l'eau de janvier 1992.

"L'eau fait partie du patrimoine commun de la nation. Sa protection, sa mise en valeur et le développement de la ressource utilisable, dans le respect des équilibres naturels, sont d'intérêt général.

L'usage de l'eau appartient à tous dans le cadre des lois et règlements ainsi que des droits antérieurement établis ".

L'eau ne peut donc faire l'objet d'une appropriation individuelle : son usage est également accessible à chacun, dans le respect des contraintes d'une exploitation collective qui prime sur les intérêts particuliers.

Dans ce cadre, seule une gestion à caractère d'intérêt général peut être mise en œuvre dont les bases fondamentales s'inspirent des principes du service public.

Accorder la primauté à l'intérêt général pour les générations actuelles et futures ; asseoir la cohésion sociale et territoriale sur l'équité et la transparence, assurer, de façon pérenne, sans solution de continuité, un service sur lequel les usagers puissent compter et qui soit en permanence capable de prendre en compte au mieux, en terme de rapport qualité/coût, l'évolution, dans le temps, des besoins et des contraintes : tels sont les termes de référence de l'éthique du service public, qui correspondent étroitement aux impératifs d'un développement durable.

Dans la pratique, la mise en œuvre de ces principes pour maîtriser la gestion, dans l'intérêt général, du service de l'eau, implique l'adhésion et la responsabilisation de tous les acteurs, et l'exercice de la clairvoyance et de l'autorité nécessaires pour les faire respecter dans la durée.

Les termes de référence du projet Canal de Provence, sa réalisation et son exploitation au quotidien se sont inspirés de ces principes et s'attachent à les respecter.

L'aménagement à buts multiples étudié, réalisé et, aujourd'hui exploité, répond à la fois par les options techniques et financières retenues aux objectifs suivants :

- satisfaire les attentes de l'utilisateur dans des conditions économiques réalistes, dans le respect des règles du service public : qualité, continuité, équité, durabilité, mutabilité et transparence,

- préserver durablement l'environnement en assurant, notamment, la gestion la plus économe de la ressource en eau et en préservant sa qualité. 
Les principaux choix effectués s'articulent autour de quelques notions simples :

- Les usagers souhaitent disposer de l'eau exactement quand ils en ont besoin, il faut donc assurer une livraison en libre service à la demande.

- La ressource en eau est limitée, il faut donc veiller à en être économe à tous les niveaux.

- L'eau du Verdon, source de l'aménagement, est de grande qualité, son transport et sa distribution doivent préserver sa qualité et respecter l'environnement.

- Et dans le respect de ces contraintes le coût global d'aménagement (investissement et exploitation) doit être le moins cher possible, afin de permettre la valorisation optimale de l'eau par l'usager.

A partir de ces quelques considérations et des principaux principes ci-après développés, les ouvrages se sont progressivement mis en place.

\section{VI ■ UN AMÉNAGEMENT À BUTS MULTIPLES : SOUPLESSE ET ADAPTABILITÉ}

La réalisation de l'aménagement à buts multiples a permis de concilier les différents usages et de bénéficier de l'économie d'échelle, confortée par la non simultanéité des pointes de demandes, au niveau des coûts d'investissement et de fonctionnement.

Le caractère multifonctionnel de l'aménagement apporte des avantages notables en termes de qualité de service, et de souplesse d'adaptation face aux changements structurels éventuels.

En qualité de service, les usages de l'eau qui, théoriquement, pourraient s'accommoder de conditions de desserte moins strictes (telle, par exemple, que l'irrigation, où une interruption de service de 48 heures est généralement possible sans grand dommage) bénéficient de fait d'une qualité de fonctionnement très supérieure, du fait des exigences plus fortes des usages urbains et industriels.

En souplesse d'adaptation aux changements structurels, il est intéressant de noter que l'ordre de grandeur des débits à transporter pour desservir un hectare n'est pas significativement différent, qu'il s'agisse d'agriculture, d'espaces verts, de zones urbaines, ou d'activités industrielles (ce qui diffère davantage, c'est le temps d'utilisation annuel de ces débits appelés de façon très saisonnière pour l'irrigation et pratiquement continue pour l'industrie).

Quelle que soit, sur le long terme, l'inévitable évolution de l'occupation des sols, en fonction de l'évolution relative des types d'activités et du mode de vie, l'adaptation des dispositifs d'acheminement de l'eau en est donc facilitée, dans la mesure où l'espace raisonnablement aménageable, à terme, est assez bien délimité.

\section{VII $\square$ DESSERTE DE L'EAU SOUS PRESSION EN LIBRE SERVICE : QUALITÉ DE SER- VICE ET PERFORMANCE}

La SCP dispose d'une capacité de réserve importante $\left(250 \mathrm{Mm}^{3}\right)$ qui l'autorise à prélever jusqu'à $660 \mathrm{Mm}^{3}$ par an dans le Verdon. Les réserves ont été créées conjointement avec EDF qui réalisait parallèlement l'équipement énergétique de la rivière. Elles sont gérées au mieux de l'intérêt collectif dans le cadre d'une convention établie dès l'origine en 1963.
Entre la prise sur le Verdon et les réseaux de distribution à l'aval, $210 \mathrm{~km}$ de gros ouvrages de transfert, capables en tête d'un débit de $40 \mathrm{~m}^{3} / \mathrm{s}$, ont été réalisés. Les $2 / 3$ du tracé sont constitués de galeries, traversant les chaînes montagneuses successives, l'autre tiers, de canaux à ciel ouvert. Le tracé a été choisi de façon à minimiser les coûts énergétiques, et limiter l'importance des pompages en tête des réseaux de distribution. Les canaux ont été revêtus et protégés des écoulements latéraux, cette solution s'avérant plus économique dans le temps et plus efficace pour éviter les pertes et protéger la qualité de l'eau. Les galeries sont, elles aussi, revêtues et fonctionnent en charge, ce qui est plus économique et d'exploitation plus facile.

Pour assurer le plus grand confort à tous les utilisateurs, industriels, communes, agriculteurs, on a choisi de livrer l'eau en libre service.

Ainsi à l'aval, la distribution d'eau a été réalisée en canalisations sous pression. Cela paraît évident pour desservir les industriels ou les communes qui reçoivent l'eau dans leurs réservoirs ou leurs installations de traitement. Mais ce l'était beaucoup moins pour l'irrigation car, jusqu'en 1955, seules les techniques d'irrigation gravitaire étaient utilisées avec une distribution au tour d'arrosage selon un calendrier rigoureux et rigide établi pour toute la saison. La SCP a décidé d'établir des réseaux distribuant l'eau sous pression, en libre service, les communes, industriels et les agriculteurs prenant l'eau quand ils le souhaitaient dans la limite du débit qu'ils ont librement souscrit, les volumes consommés étant mesurés par un compteur. Tous les utilisateurs peuvent ainsi consommer à tout moment toute l'eau dont ils ont besoin.

\section{VIII — PRÉSERVATION QUANTITATIVE ET QUALITATIVE DE LA RESSOURCE : UNE PRÉOCCUPATION PERMANENTE, DE LA RÉALISATION À L'EXPLOITATION}

Comme la ressource en eau est limitée, une politique systématique de gestion économe de la ressource a été appliquée dans un souci d'éviter tout risque d'hypothéquer l'avenir en laissant s'établir des comportements de gaspillage.

Dans le cas de cet aménagement, tout gaspillage d'eau représente, en effet, une perte économique sensible :

- au niveau d'ensemble de la collectivité, parce que cette eau gaspillée aurait pu trouver une autre valorisation (énergétique notamment) au lieu d'être indûment prélevée,

- au niveau de l'aménagement proprement dit, parce qu'il induit des coûts supplémentaires d'investissement et de fonctionnement,

- au niveau de l'utilisateur, dans la mesure où la tarification lui répercute le coût de son gaspillage.

Ces considérations ont guidé la conception technique des ouvrages : tracé optimisé, galeries en charge, canaux revêtus, distribution par conduites sous pression ; invention, dès 1971 , du système de régulation dynamique du mouvement de l'eau dans les ouvrages de transport, qui ne sont sollicités qu'à hauteur des besoins à satisfaire - ni plus, ni moins - d'où économie d'investissement et absence totale de perte d'eau par déversement ; télésurveillance en continu de la qualité de l'eau.

Elles ont guidé également la programmation des investissements, modulée en fonction de l'évolution réelle de la demande. 
Elles sont toujours présentes au quotidien dans le mode de gestion technique des ouvrages, qui s'appuie sur un ensemble cohérent de mesures disponibles en temps réel, systématiquement exploitées pour s'assurer de l'évolution du comportement et des performances des ouvrages et aussi du maintien de leur adaptation aux besoins des utilisateurs.

Elles ont enfin fondamentalement guidé le choix du système de tarification au coût marginal de développement à long terme.

\section{IX @ UNE TARIFICATION AU SERVICE DE L'INTÉRÊT GÉNÉRAL}

La tarification adoptée est basée sur les principes de tarification au coût marginal de développement, dont la théorie économique indique qu'elle oriente les décisions des consommateurs dans le sens de l'intérêt général. Elle vise en effet à répercuter à chaque client les charges qu'il faut supporter pour lui fournir un débit d'un mètre cube/heure supplémentaire ou encore un volume d'un mètre cube supplémentaire en période de pointe (en été) ou en période hors pointe (en hiver).

Elle est donc diversifiée en fonction du mode d'utilisation par le client des débits mis à sa disposition, ce qui l'incite à souscrire au mieux de son intérêt et à ne pas consommer inconsidérément.

Cette théorie tarifaire permet de répartir de manière équitable et objective les charges entre les diverses utilisations de l'eau, urbaine, industrielle ou d'irrigation. Elle fournit un repère vis-à-vis de la situation d'optimum économique. Il n'est pas interdit de s'en écarter, mais au moins sait-on de combien on s'en écarte.

Dans la pratique la SCP s'est efforcée d'arriver à des tarifs réalistes, clairs et maniables. Les tarifs pratiqués sont des tarifs binômes avec :

- une redevance de débit,

- et une redevance de consommation.

La redevance de débit correspond aux dépenses qu'il faut consentir pour tenir à la disposition immédiate du consommateur le débit qu'il a souscrit. Cette redevance est fonction directe des débits souscrits et d'autant plus élevée que l'on s'éloigne du point de prélèvement. Trois zones tarifaires ont été établies. Pour des conditions identiques de consommation, l'influence de la zone tarifaire joue de la façon suivante :

$\begin{array}{lll}- \text { zone I : } & 1,35 \\ - \text { zone II }: & 1,00 \\ - \text { zone III }: & 0,55\end{array}$

Ce système s'applique à tous les usages en fonction de leurs caractéristiques propres. Quelques aménagements ont progressivement été apportés :
- les agriculteurs de profession bénéficient d'un abattement de $60 \%$ sur le tarif d'irrigation, marquant ainsi la solidarité effective des divers secteurs d'activité en faveur de l'agriculture dans le cadre d'une politique d'aménagement du territoire,

- pour les usagers urbains et industriels, des tarifs d'utilisation saisonnière et d'utilisation en secours ont été développés pour faire face à deux types de situation. Soit il s'agit des communes du littoral qui voient leur population s'accroître considérablement en période estivale, soit il s'agit de clients qui disposent d'une ressource propre mais qui souhaitent disposer de moyens leur permettant de faire face à une défaillance imprévue et limitée dans le temps de cette res. source.

Aujourd'hui, cette forme de tarification donne tout à fait satisfaction, compte tenu des ajustements et des compléments successifs rendus nécessaires par les demandes diversifiées des usagers.

Les tarifs ont été indexés sur les indices économiques représentatifs des coûts mis en œuvre et leur évolution reflète effectivement l'évolution des charges de toute nature que supporte la société.

\section{X — UNE GESTION ÉCONOME ET ÉQUILIBRÉE}

Grâce à la conduite d'une politique globale, intégrée et cohérente de gestion économe de la ressource en eau dont quelques termes de références ont été abordés ci-dessus, la Société du Canal de Provence assure continûment depuis 40 ans le service de l'eau.

Guidée par des principes de gestion durable d'un service public, la SCP a contribué à mettre une grande partie du territoire provençal à l'abri de la pénurie d'eau et permis l'aménagement de l'espace régional et de développement de ses activités économiques.

Le travail collectif entrepris au niveau régional a permis d'atteindre un équilibre malgré les objectifs initiaux ambitieux. Le prix de l'eau payé par les consommateurs, intègre la valeur de l'eau dans son site et couvre aujourd'hui intégralement le coût du service, à savoir les charges de gestion, d'exploitation, de maintenance et de renouvellement, ainsi que les charges financières de remboursement des emprunts. Le maintien durable d'un tel équilibre nécessite un effort constant au quotidien pour s'adapter à la réalité économique et améliorer en permanence les performances.

La triple compétence d'une société d'aménagement régional comme la SCP, concepteur-réalisateur-gestionnaire, l'aide incontestablement à optimiser la conduite des investissements en tenant compte des coûts récurrents de leur exploitation pour satisfaire dans les meilleures conditions et en équité ses usagers dans le cadre d'une vision d'aménagement durable préservant le patrimoine des ressources en eau. 